\title{
WestVirginiaUniversity
}

THE RESEARCH REPOSITORY @ WVU

Graduate Theses, Dissertations, and Problem Reports

2018

\section{Efficient wound assessment system with an RGB-D camera}

Xiang Gao

Follow this and additional works at: https://researchrepository.wvu.edu/etd

\section{Recommended Citation}

Gao, Xiang, "Efficient wound assessment system with an RGB-D camera" (2018). Graduate Theses, Dissertations, and Problem Reports. 3989.

https://researchrepository.wvu.edu/etd/3989

This Problem/Project Report is protected by copyright and/or related rights. It has been brought to you by the The Research Repository @WVU with permission from the rights-holder(s). You are free to use this Problem/Project Report in any way that is permitted by the copyright and related rights legislation that applies to your use. For other uses you must obtain permission from the rights-holder(s) directly, unless additional rights are indicated by a Creative Commons license in the record and/ or on the work itself. This Problem/Project Report has been accepted for inclusion in WVU Graduate Theses, Dissertations, and Problem Reports collection by an authorized administrator of The Research Repository @ WVU. For more information, please contact researchrepository@mail.wvu.edu. 


\title{
Efficient Wound Assessment System with an RGB-D Camera
}

\author{
Xiang Gao
}
Problem Report submitted
to the Benjamin M. Statler College of Engineering and Mineral Resources at West Virginia University

in partial fulfillment of the requirements for the degree of

\author{
Master of Science in \\ Computer Science \\ Victor Fragoso, Ph.D., Chair \\ YanFang Ye, Ph.D. \\ Saiph Savage, Ph.D.
}

Lane Department of Computer Science and Electrical Engineering

Morgantown, West Virginia

2018

Keywords: RANSAC, Kinect, wound assessment, rigid transformation, morphological transformation, dilation, thinning, fitting plane, point cloud CCopyright 2018 Xiang Gao 


\section{ABSTRACT \\ Efficient Wound Assessment System with an RGB-D \\ Camera}

\section{Xiang Gao}

Continuous monitoring of changes in wound size, wound area, and volume, is key to predict whether wounds will heal on time. Wound measurement methods can be subdivided into non- contact and contact methods. Contact methods are prone to errors given the human intervention and it increases the chance of discomfort during measurement. Alternative methods, such as image- based non-contact methods, eliminate any discomfort and have good reliability for measuring a wound. However, existing image-based non-contact methods are expensive. This is because these methods build a 3D model of the wound using expensive devices in order to allow the clinician to obtain the necessary wound measurements. To alleviate the cost of these systems, the proposed system described in this report measures wounds using lowcost depth cameras such as the Microsoft Kinect. This report describes methods that take in an RGB image from the Microsoft Kinect, computes the necessary parts of a 3D wound model, and finally reports wound measurements. The proposed system requires the user to draw the contour of the wound on the image. Then, the system automatically extracts all the necessary information from the RGB and depth images to create a minimal 3D model of the wound. Subsequently, the system processes the 3D model in order to facilitate the estimation of the wound area and volume. Finally, the system reports the measurements to the user. This report presents experiments demonstrating that the proposed system achieves acceptable measurements despite the fact that it uses a low-cost and noisy imaging sensor. 


\section{Acknowledgements}

I would like to express my deep gratitude to Dr. Victor Fragoso. I'm truly grateful of his patience, motivation, enthusiasm, and immense knowledge. His constant support guided me through the research. Besides my advisor, I would like to thank all my colleagues. I enjoyed the discussion with them when I had issues with our research.

I would like to extend my thanks to the rest of my committee members: Prof. YanFang Ye and Prof. Saiph Savage, for being my committee member, reading my report and coming to my defense.

Last but not least, I wish to thank my family who always has supported me, my parents Qitian Gao, Chunhua Wang, and most of all Yue Ren for enjoying life together with me. 


\section{Table of Contents}

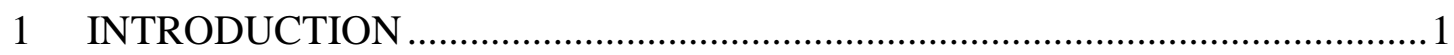

2 QUICK WOUND ASSESSMENT APPROACH .............................................

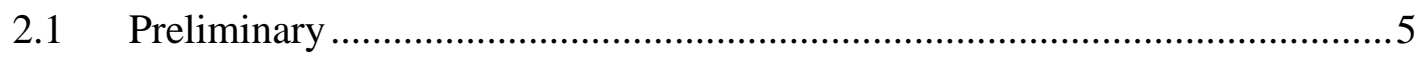

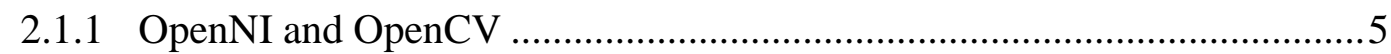

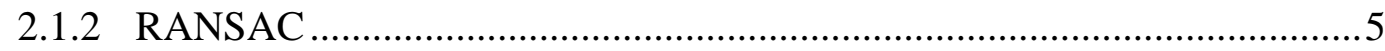

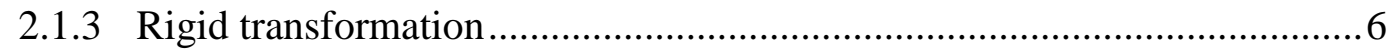

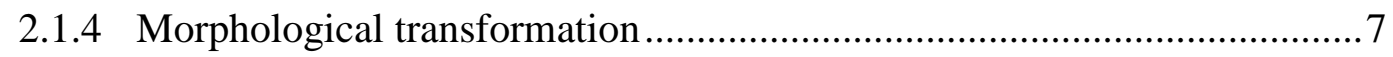

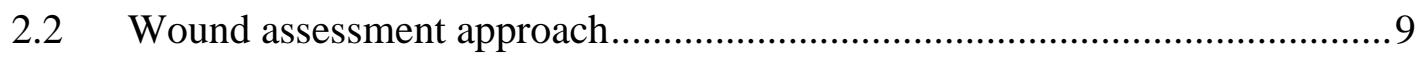

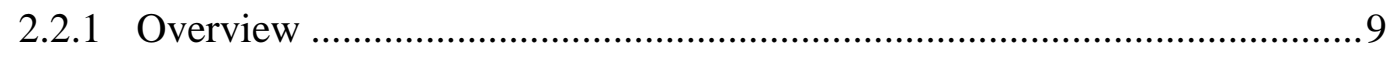

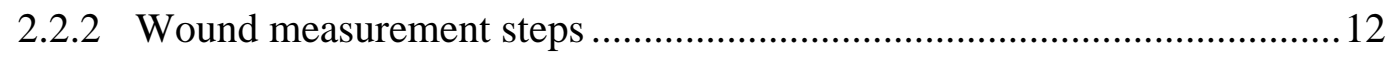

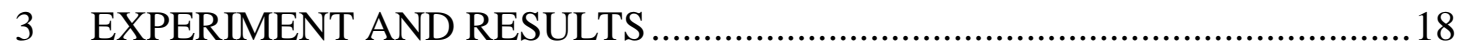

3.1 Experiment 1: Fitting plane verification …................................................ 19

3.2 Experiment 2: Rigid transformation verification ...................................25

3.3 Experiment 3: Morphological transformation verification .........................25

3.4 Experiment 4: Robustness and measurement accuracy verification .............27

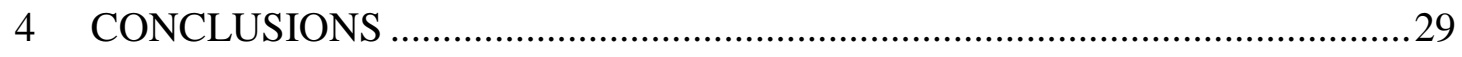

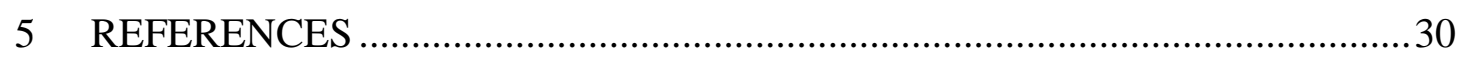




\section{List of Figures}

Figure 1: Wound Measurement: Ruler Method. ......................................................2

Figure 2: Wound Measurement: Tracing Method. ..................................................2

Figure 3: The 3D coordinate system built in the system and the equation for rigid transformation.

Figure 4: Overview of the proposed approach...................................................... 10

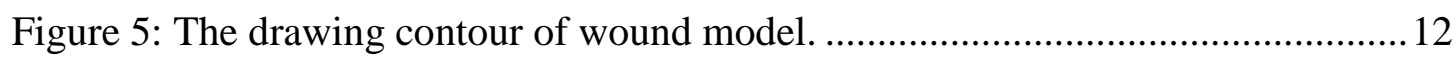

Figure 6: Definition and equation used in RANSAC. …....................................... 14

Figure 7: Paper model (left) and wound model (right)..........................................18

Figure 8: The outline wound points and the fitting plane for the paper model. ..........20

Figure 9: The histogram of the distance of the outline points to the fitting plane.......20

Figure 10: The fitting plane and the point cloud of the wound model. ......................23

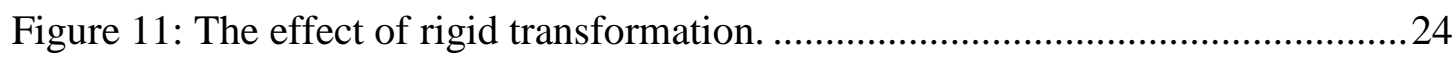

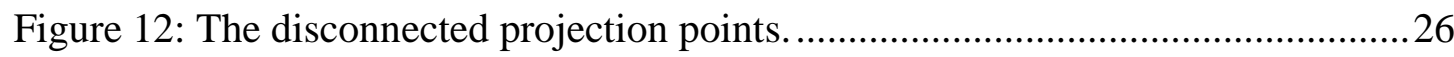

Figure 13: The effect of dilation and thinning operation........................................26 


\section{List of Tables}

Table 1: The standard deviation of the distance of mapped border points to the fitting plane and the ratio of inlier points of the fitting plane for the wound model. 21

Table 2: The average and standard deviation of the output volume and area for the

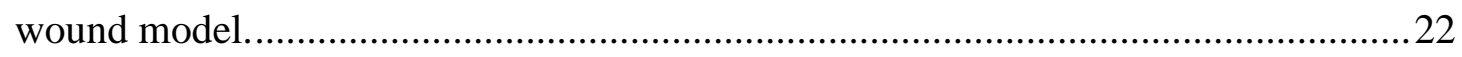

Table 3: The area measurement for paper model..................................................28 


\section{INTRODUCTION}

Frequent wound measurement is an important part of wound care. Continuous monitoring of changes in wound size, wound area, and volume is key to predict whether wounds will heal on time with a conventional treatment and deciding which patients are candidates for often expensive new treatments [1][2]. For example, a percentage change in wound area over a four-week period of $30 \%$ or more is a good predictor of healing in venous leg ulcers and diabetic foot ulcers [2][3].

Wound measurement methods can be subdivided into non-contact and contact methods, like ruler and tracing method. Contact methods give a rough estimate of the total wound area and the evaluation depends on human experience. As such, these methods are prone to errors given the human intervention in the measuring process and increase the chances of non-sanitary complications given the physical contact between measuring objects. Consequently, these methods may not be good for validating the healing process [4]. Alternative methods, such as, image-based methods which are non-contact methods, are more reliable because of their computing power and sensor technologies but may require expensive sensors.

The ruler-based method is a quick, easy, and inexpensive procedure to measure the length and width of a wound; see Fig.1. The most common one is to measure the longest length and width, regardless of head-to-toe orientation, or to measure length and width perpendicular to one another [5]. After obtaining wound length and width, the clinician multiplies the 2 measurements to obtain the area. Therefore, the estimated area will literally always be greater than the actual area, up to $44 \%$ [3]. This was shown to be true especially for a pear-shaped wound. Methods such 


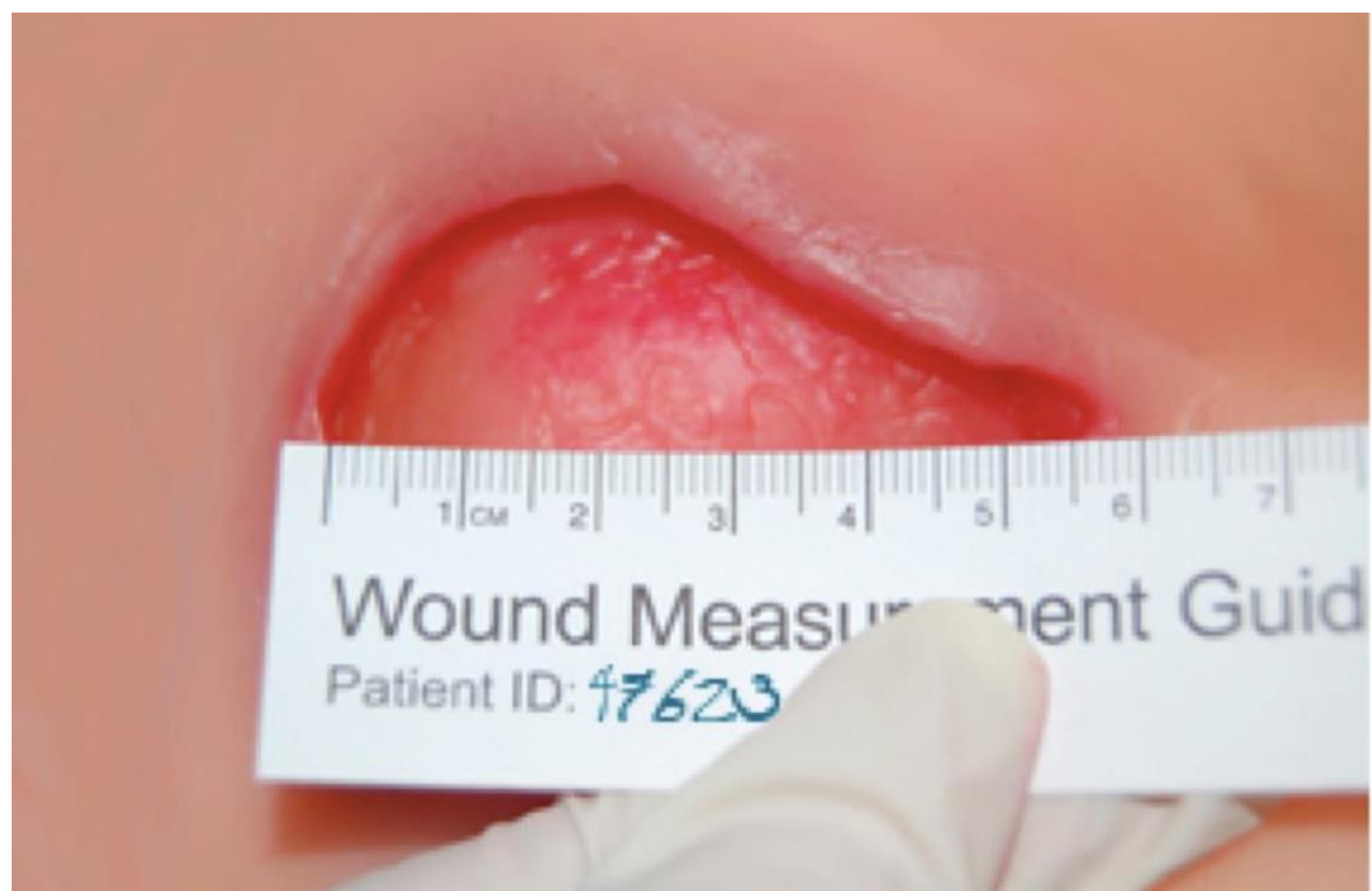

Figure 1: Wound Measurement: Ruler Method. Clinicians place a disposable ruler close to the wound to measure the length and width of the wound.

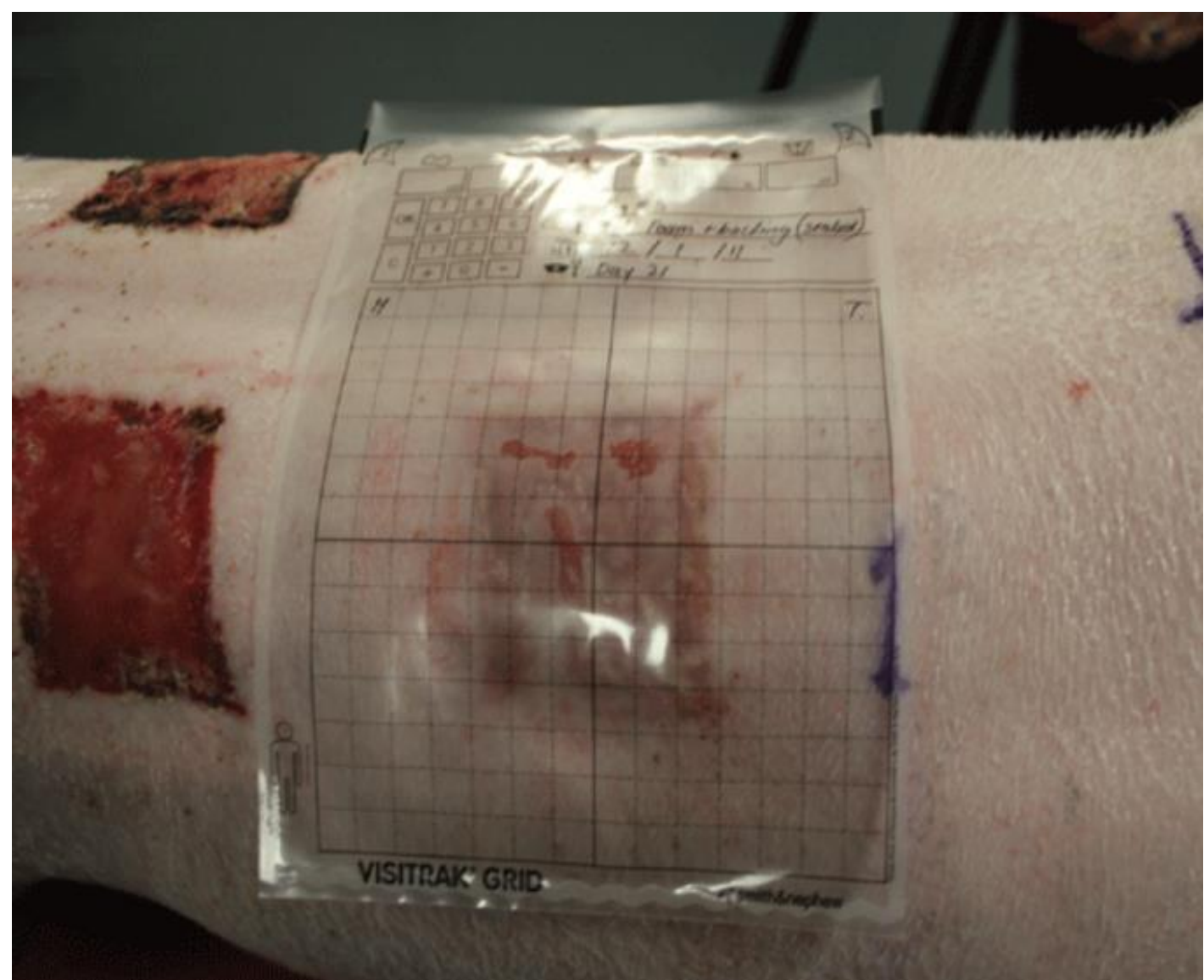

Figure 2: Wound Measurement: Tracing Method [6]. Clinicians place a tracing paper or film onto the wound to trace the wound boundary with a pen. 
as the ruler technique are inaccurate and do not account for changes in wound shape and such practice should be discouraged.

In the tracing method, the clinician traces the wound perimeter onto a transparent acetate with a permanent pen; see Fig.2. Some acetates are preprinted in $1 \mathrm{~mm}^{2}$ or 1 $\mathrm{cm}^{2}$ grid. The area is calculated by counting the number of squares within the wound perimeter. Although this is a relatively easy method with a good reliability, the tracing process can cause some discomfort [5], and it may take time to count the squares [3].

There has been a significant progress in imaging devices. These devices present various advantages, and in particular they are easy to use. As such, these devices can be useful to eliminate any discomfort and good reliability for measuring a wound. For instance, a video device can be used to record the optical characteristics of the wound. Based on the input of the digital sensor, a 3D model of the wound can be built using 3D computer vision techniques [7]. Morphometric analysis of the model enables the clinician to obtain the geometric determination of the perimeter, area, and maximum vertical and horizontal dimensions in a relatively simple and precise way. To generate more accurate measurements, we can use dense 3D models of the wound. To do so, laser-assisted devices are often used and commercially available, e.g., the SilhouetteStar with a $\$ 8,000$ price. Unfortunately, these devices are expensive and are not easy to use. Fortunately, the emergence of low cost depth cameras, e.g., the Microsoft Kinect, provides an alternative way to generate these 3D wound models. By using these cameras, it is possible to perform the wound assessment more convenient and accessible to all clinicians.

The proposed system described in this report aims to measure wound metrics using low-cost depth cameras such as the Microsoft Kinect. This report describes 
methods that take in an RGB image and a depth image from the Microsoft Kinect, computes the necessary parts of a 3D-wound model, and finally reports wound measurements. The infrared (IR) light projector mounted on the Kinect emits structured light and it is received by another sensor also in the Kinect. The depth information can be inferred by analyzing of the deformed light signal. Compared to most commercial available 3D scanners, the Kinect sensor is affordable. Although the Kinect depth information can be considered noisy and imperfect [8], many researchers and companies use it as a 3D modeling tool and obtain compelling results [9][10][11]. It is possible to obtain a point cloud from the Kinect sensed information. This point cloud contains the $\mathrm{X}, \mathrm{Y}$, and $\mathrm{Z}$ coordinates of every point in the scene, which are useful to represent the sets of points on the surface of objects in a three-dimensional coordinate system. This point cloud can be used to generate 3D meshes or dense 3D model for our purpose. 


\section{QUICK WOUND ASSESSMENT APPROACH}

\subsection{Preliminary}

The proposed approach quickly estimates wound assessment parameters, like the area and volume. The approach consists of a series of operations, and the algorithm utilizes several libraries and algorithms, which are explained first to help the reader better understand the approach.

\subsubsection{OpenNI and OpenCV}

The proposed system utilizes Microsoft Kinect to capture 3D information of the wound. To acquire color and depth images of the wound, the approach uses OpenNI ${ }^{1}$ (Open Natural Interaction), which is an open source multi-language, cross-platform framework and the driver for Microsoft Kinect to enable the data acquisition and communication with the RGB and depth cameras of the Kinect. The image grabbed from the Kinect and created by the system can be easily processed with the help of OpenCV ${ }^{2}$, which is an open source library that provides support for different applications in computer vision. The proposed system utilizes OpenCV to process the image.

\subsubsection{RANSAC}

Plane fitting is a prerequisite to many kinds of tasks. To calculate the area of the wound, the proposed system fits a plane in 3D. To do so, the approach utilizes Random Sample and Consensus (RANSAC). This algorithm was proposed by

\footnotetext{
${ }^{1}$ https://github.com/OpenNI/OpenNI

${ }^{2}$ https://opencv.org/
} 
Fischler and Bolles [12] and is widely used in many applications in Computer Vision. It is a general model parameter estimation approach and widely adopted by the computer vision community. RANSAC uses a very small portion of input data (minimum number observations to build a model) to generate enough candidate solutions [13]. RANSAC evaluates every potential solution using the entire data set and chooses the best solution at last. The most famous advantage of this method is that it can deal with data that contains a large proportion of outliers, which are data points that deviate from an underlying model. Different from traditional sampling techniques, which use all the input data at once to simulate the initial model followed by heavily mathematical optimization, RANSAC is an iterative method that randomly uses smallest sets repeatedly to generate possible solutions followed by an evaluation phase. Although outliers can influence some of the candidate solutions, it is guaranteed that there exists a potential solution free of outlier influence. Since there is no underlying consistency in the outliers, the potential solution free of outlier influence has a better evaluation with respect a model. Although RANSAC algorithm is widely used for plane detection [12], especially in 2D images, the system utilizes RANSAC in order to fit a plane in 3D [14][15][16].

\subsubsection{Rigid transformation}

The proposed system builds two 3D coordinate systems to transforms points between them to facilitate the assessment. One is the default coordinate system of the Microsoft Kinect, the other is obtained by rotating the default one. Any 3D point can be represented in the two coordinate systems and transformed to each other through rigid transformation; a transformation that does not alter the relative position of all the points is called rigid transformation. Translations, rotations, and combinations of these transformations are rigid transformations. As illustrated in Fig.3 and based on 
the Equation 1, the same point represented by $\left(\mu_{1}, \mu_{2}, \mu_{3}\right)$ in $x_{1}, x_{2}$ and $x_{3}$ coordinate system is transformed to representation $\left(\mu_{1}^{\prime}, \mu_{2}^{\prime}, \mu_{3}^{\prime}\right)$ in the new $x_{1}^{\prime}, x_{2}^{\prime}$ and $x_{3}^{\prime}$ coordinate system. Since it is a rigid transformation, the relative position of a set of points will be preserved. During assessment, the system exploits rigid transformation to transform points between the two 3D coordinate systems to facilitate the assessment.

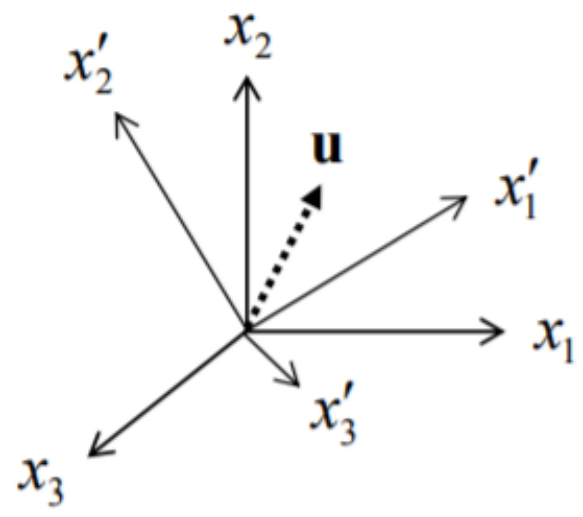

Figure 3: The 3D coordinate system built in the system and the equation for rigid transformation [17]. $x_{1}, x_{2}, x_{3}$ are the basis vectors of the default coordinate systems and $x_{1}^{\prime}$, $x_{2}^{\prime}, x_{3}^{\prime}$ are the basis vectors of the other coordinate systems. $\cos \left(x_{i}^{\prime}, x_{j}\right)$ is the cosine of the angle between the $x_{i}^{\prime}$ and $x_{j}$.

$$
\left[\begin{array}{l}
\mu_{1}^{\prime} \\
\mu_{2}^{\prime} \\
\mu_{3}^{\prime}
\end{array}\right]=\left[\begin{array}{lll}
\cos \left(x_{1}^{\prime}, x_{1}\right) & \cos \left(x_{1}^{\prime}, x_{2}\right) & \cos \left(x_{1}^{\prime}, x_{3}\right) \\
\cos \left(x_{2}^{\prime}, x_{1}\right) & \cos \left(x_{2}^{\prime}, x_{2}\right) & \cos \left(x_{2}^{\prime}, x_{3}\right) \\
\cos \left(x_{3}^{\prime}, x_{1}\right) & \cos \left(x_{3}^{\prime}, x_{2}\right) & \cos \left(x_{3}^{\prime}, x_{3}\right)
\end{array}\right]\left[\begin{array}{l}
\mu_{1} \\
\mu_{2} \\
\mu_{3}
\end{array}\right]
$$

Equation 1: The rigid transformation equation. This equation does rotation transformation between two coordinate systems.

\subsubsection{Morphological transformation}

To assess the wound, the approach places sparsely distributed data points in a metric image. The area encompassed by these points is the wound area. Then the system connects these points through morphological transformation to form a closed 
circle to facilitate further measurement. Morphological transformations are simple operations related to the shape or morphology of features in an image. It is normally performed on binary images with structuring element; a structuring element is a small matrix with a small shape or template, which decides the nature of operation. Morphological operations are applied by convolving a binary image and the structuring element positioned at all possible locations in the image. The two-main basic morphological operators are dilation and erosion.

Dilation is the morphological operation that corresponds to the intuitive concept of "growing" by adding a layer of pixels around the small structures. After dilation, small structures become bigger and discrete sparse structures may become connected because of the addition of pixels in the gap between the disconnected structures. The proposed system performs dilation on the wound image to connect discrete pixels.

Erosion is the opposite of dilation. Structures in the image are peeled off a layer of a certain thickness around the boundaries, which aligns with the intuition of "shrinking". Erosion removes a layer of pixels from the borders of structures. After erosion, smaller structures may disappear and only the larger structures remain. Thinning is another common morphological operation, which is very similar to erosion. By applying iterative "conditional" erosion, thinning shrinks binary structures down to a minimum thickness of one pixel without splitting the structure into multiple parts [18]. Through checking the local neighborhood for every image position, thinning decides to apply another erosion step or not at this position. Zhang and Suen thinning algorithm [19] is one classic thinning algorithm, which defines two boolean predictors based on how many neighbors around the central position and how many components are connected via the current center position, so-called "connectivity number". The two predictors indicate whether another erosion operation 
will shrink the thick structure or cause a separation to occur. Depending on the outcome of the two predictors, the pixel is either deleted or marked as non-removable. At every iteration, only one-layer of pixels are selectively deleted. The thinning operation continues until no more changes are needed. After a dilation operation connects the disperse pixels in the image, the system performs thinning operation to obtain the skeleton of the now connected structures.

\subsection{Wound assessment approach}

\subsubsection{Overview}

To assess the wound, the proposed system captures a 3D image of the wound using the Microsoft Kinect, which provides color and depth images. Through careful calibration and with the use of external libraries, such as OpenNI and OpenCV, the system perfectly aligns the depth and color images. A workflow of the system is presented in Fig. 4. The first step in the system requires the user to draw the contour of the wound on the color image following the wound boundary. Next, the system maps every pixel along the contour of the wound to a point in the depth map, which is a 2D image with depth values at pixel positions. This is possible thanks to the calibration parameters discussed earlier. Consequently, for every pixel we can get a point in the 3D space. Under the hypothesis that the selected skin region is nearly flat in the healthy region, the mapped border points of the wound should fall on a 3D plane. Then, the system utilizes RANSAC algorithm to obtain the fitting plane with the mapped points. Due to noise, the mapped border points won't perfectly fall onto the plane. The system projects the entire mapped border points in the $3 \mathrm{D}$ space onto the fitting plane and stores these projected points. Further, the system builds a new coordinate system using the normal vector of the fitting plane as the $\mathrm{Z}$ axis of the new 
coordinate system. This is to ensure that the projected points have the same $\mathrm{Z}$ coordinate in the new coordinate system. Through a rigid transformation, the approach further transforms the projected points from the default coordinate system to

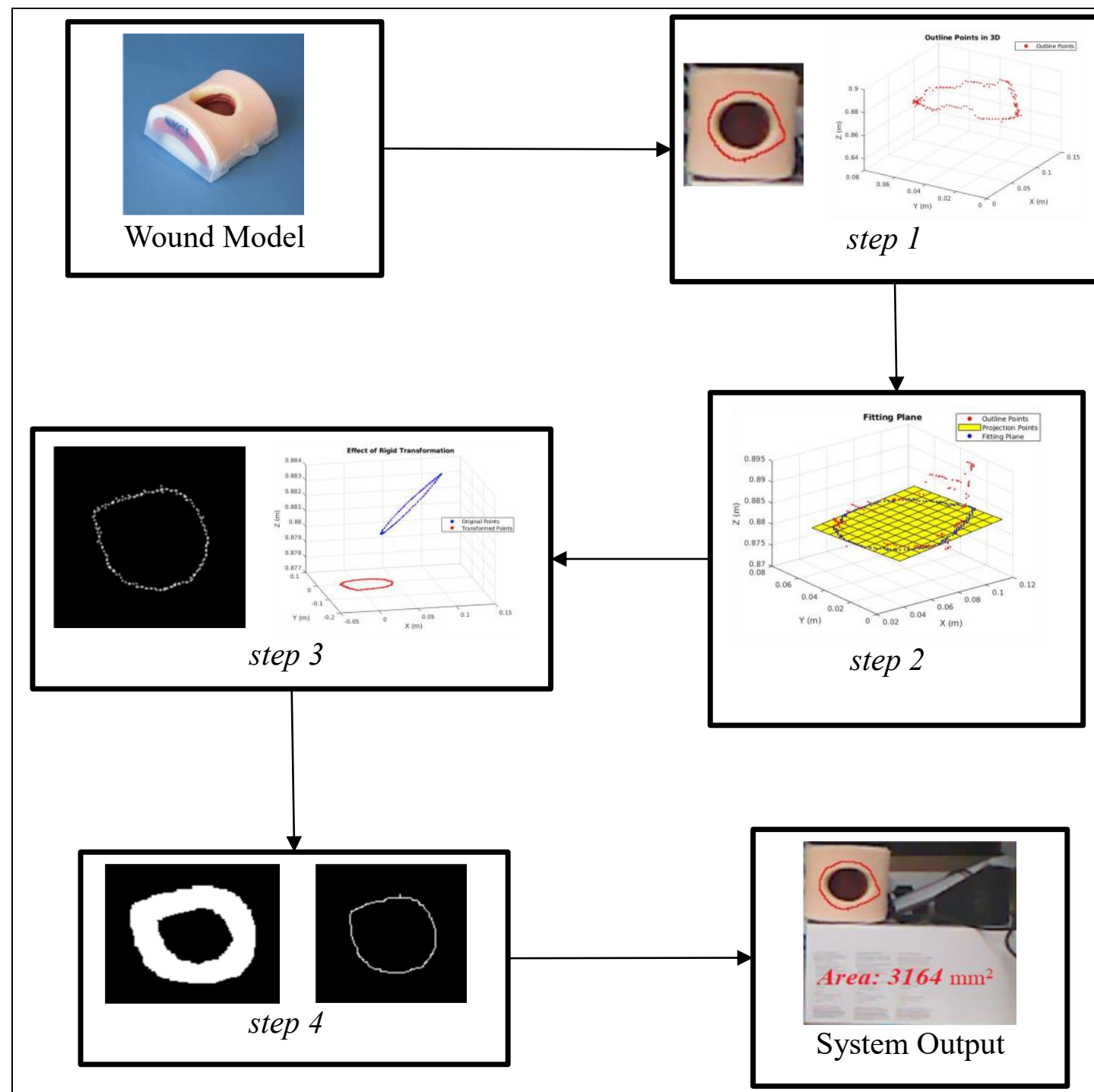

Figure 4: Overview of the proposed approach. The wound is placed within the field of view of the Kinect. Step 1: Outlining the wound and mapping the wound outline into the point cloud. Step 2: Estimating a plane that fit the points of the wound and obtain the projection points. Step3: Mapping the 3D project points of the would onto a 2D plane through rigid transformation. Step 4: Placing the points in a metrics image followed by dilation and thinning operation to get the skeleton of the boundary. Finally, the system outputs the calculated area or volume.

the new built one while preserving relative position. By ignoring the same $\mathrm{Z}$ value of the transformed points in the new coordinate system, the proposed approach transforms the $3 \mathrm{D}$ projected points to a $2 \mathrm{D}$ plane. Then the system places the points in 
an image using the $\mathrm{X}, \mathrm{Y}$ coordinates of the points as the positions of the points in the image. With the help of morphologic transformations, the system performs dilation and thinning operations on the image to get the skeleton of these points, which is a closed contour. The system calculates the wound area by counting the pixels within the contour with the help of OpenCV. At the same time, the approach can get the perimeter and bounding box of the contour. Wound assessment parameters, length and width, are the length and width of the bounding box, encapsulating the wound boundary. At this stage, the system successfully estimates the length, width, and area of the wound.

The system follows the similar process to calculate the volume of the wound. Although the system only utilizes the outline of the wound border to calculate the wound area, the proposed approach uses the entire wound region to calculate the volume. The region of interest on the color and depth image can also be transformed into 3D space where the metric measurement is performed. Since the wound region is composed of pixels from both the color and depth images, the system first partitions the wound cavity into small cubic based on the pixels. For each pixel, there is one corresponding point in the 3D space and the proposed approach maps the pixel itself and three neighbors (the right, lower and the lower right pixels of this pixel) into 3D space to get four corresponding points and further projects these four mapped points onto the fitting plane to get four projection points, which form a quadrilateral. The system iterates all the pixels and sums up the area of all the quadrilaterals as the area of the wound region, which is another way to calculate the wound area. The four mapped points in the 3D space and the four projection points on the fitting plane form a cubic. The system iterates all the pixels and sums up the volume of all the cubic as the volume of the wound. 
In general, the approach consists of five steps: 1) mapping the wound outline into the point cloud; 2) estimating a plane that fits the points of the wound; 3) mapping the $3 \mathrm{D}$ points of the wound onto the estimated $2 \mathrm{D}$ plane; 4) measuring the wound area and; 5) volume, which are fully explained below and illustrated in Fig. 4.

\subsubsection{Wound measurement steps}

Map the wound outline into the point cloud (step 1): The wound, which is about to be assessed, is monitored by the Kinect to obtain the wound's 3D information. First, the system grabs the color image, depth image, and the point cloud containing wound information from the Kinect. On the color image, which is displayed on a screen of a mobile device such as phone or tablet, the operator draws a contour along the wound boundary; see Fig.5. At the same time, the

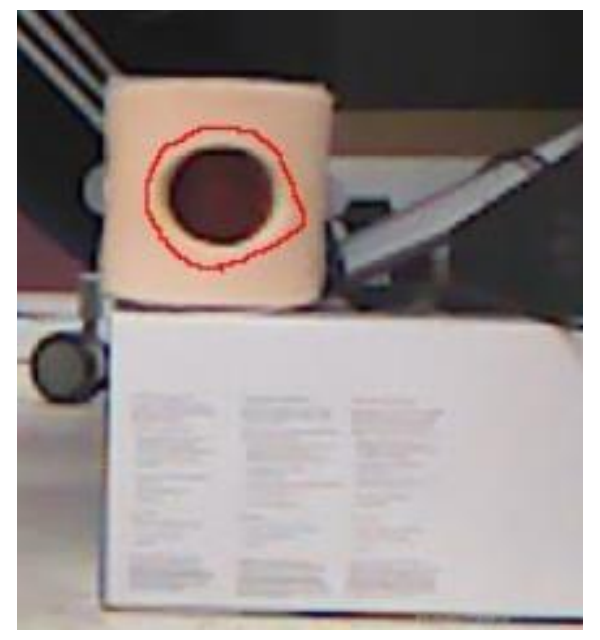

Figure 5: The drawing contour of wound model. The red contour is drawn on the color image with a mouse. system records the coordinates of each pixel along the contour. Once the contour is finished, the system automatically connects the head and tail of the contour to form a closed contour. Because the depth and color images, and point cloud are captured by the Kinect, every point in the scene has one corresponding pixel in the color and depth images. Each pixel in the color image can be mapped to another corresponding pixel in the depth image through a series of calculations based on the multiview geometry theory [7]. With the help of the OpenNI library, the proposed approach maps easily the recorded contour pixels in the color image to the corresponding pixels in the depth image through the OpenNI registration process. Once registered, it is easy to obtain the 3D point in the point cloud. Because 
the point cloud is the sparse 3D model of the scene, some points in the scene will not have perfect corresponding points in the point cloud. But every point in the scene can be mapped to one point in the point cloud, which is closest to the real corresponding point in the 3D space. Thanks to the OpenNI library, the system successfully maps recorded pixels of the contour in the color image to points in the point cloud. At this point, the system maps wound outline pixels in the $2 \mathrm{D}$ image to points in the $3 \mathrm{D}$ space with $\mathrm{X}, \mathrm{Y}$ and $\mathrm{Z}$ coordinates.

\section{Algorithm: RANSAC for plane detection}

1. Iteration $=200$, SupportMax $=0$, DistanceThreshold $=0.0064$, PlaneEquation

$$
a x+b y+c z+d=0
$$

2. For $\mathrm{i}=0$ to Iteration

3. SupportTemp $=0$

4. Find a plane by randomly choosing three points from points set

5. For $\mathrm{j}=0$ to points set size

6. Find the distance of the point $\mathrm{j}$ to the plane

7. If distance $<$ DistanceThreshold

8. Increment SupportTemp

9. $\quad$ End

10. If SupportTemp $>$ SupportMax

11. Update SupportMax, PlaneEquation

12. End

13. End

14. Output PlaneEquation

Estimate a plane that fit the points of the wound (step 2): To obtain the wound area, the proposed system first fits a plane to the 3D point cloud, and the region encompassed by the recorded 3D points on the fitted 3D plane is the wound area. The 
hypothesis is that the healthy skin is nearly flat, and it is where the fitting plane resides. Since the outline of the wound region falls on the same 3D plane, the system utilizes these outline points to fit a plane using RANSAC algorithm which is described in the Algorithm above. Some definition and steps of RANSAC are described below. As illustrated in Fig.6, RANSAC randomly chooses three points $a, b$ and $c$ from the pool of candidate 3D points from the outlined points. Then, RANSAC builds a potential model, a plane containing points $a, b$ and $c$, followed by a plane

$$
\begin{aligned}
& \overrightarrow{a b}=\left[\begin{array}{l}
b_{x}-a_{x} \\
b_{y}-a_{b} \\
b_{z}-a_{c}
\end{array}\right] \\
& \vec{x}=\overrightarrow{a b} \div|\overrightarrow{a b}| \\
& \overrightarrow{a c}=\left[\begin{array}{l}
c_{x}-a_{x} \\
c_{y}-a_{b} \\
c_{z}-a_{c}
\end{array}\right] \\
& \vec{w}=\overrightarrow{a b} \times \overrightarrow{a c} \\
& \vec{z}=\vec{w} \div|\vec{w}| \\
& \vec{y}=\vec{z} \times \vec{x} \\
& \vec{z}=\left[\begin{array}{l}
z_{x} \\
z_{y} \\
z_{z}
\end{array}\right] \\
& \vec{e}=\left[\begin{array}{l}
a_{x}-p_{x} \\
a_{y}-p_{b} \\
a_{z}-p_{c}
\end{array}\right] \\
& d=\vec{z} \cdot \vec{e} \\
& p_{\text {new }}=\left[\begin{array}{l}
p_{x}-d * z_{x} \\
p_{y}-d * z_{y} \\
p_{z}-d * z_{z}
\end{array}\right]
\end{aligned}
$$

Figure 6: Definition and equation used in RANSAC. $\left(a_{x}, a_{y}, a_{z}\right)$ is the representation of a point a. This is applied to point $\mathrm{b}, \mathrm{c}, \mathrm{p}$. The cross product of two vectors $\overrightarrow{a b}$ and $\overrightarrow{a c}$ is $\vec{w}$ and normalized to $\vec{z}$, which is the norm vector of the plane containing a, b and c. For every point $\mathrm{p}$ in the pool, new vector $\vec{e}$ is defined with $\mathrm{p}$ and a. The distance of the point $\mathrm{p}$ to the fitted plane is $\mathrm{d}$ and $p_{\text {new }}$ is the projection point of $\mathrm{p}$ onto the fitting plane. 
evaluation. For every point $p$ in the pool, RANSAC calculates the distance of the point $p$ to the fitting plane as $d$. If $d$ is less than the predefined threshold, the point $p$ supports the plane. Then, RANSAC counts the total points supporting the plane. If the new fitted plane has bigger support than the previous one, RANSAC accepts the plane, otherwise it is abandoned. After several iterations (which are predefined as a parameter) of the above process, the plane with the largest support is accepted by RANSAC as the fitting plane containing the contour points in the 3D space. Due to noise, points won't perfectly fall onto the plane. For every point $p$ in the pool, the system records the projection point $p_{\text {new }}$ for the next step.

Map the $3 D$ points of the would onto the estimated $2 D$ plane (step 3): The region encompassed by the projection points on the fitting plane is the area of the wound. But the projection points only have the coordinates based on the default $\mathrm{X}, \mathrm{Y}$ and $\mathrm{Z}$ coordinate system of the Kinect and it is not convenient to calculate the area using these coordinates. Fortunately, the proposed system builds another coordinate system, using the same origin as the default coordinate system and $\vec{x}, \vec{y}, \vec{z}$ as the three basis vectors. Since $\vec{z}$ is also the normal vector of the fitting plane, these projection points have the same $\mathrm{Z}$ coordinate in the new coordinate system. Based on the rigid transformation Equation 1, the proposed approach transforms points from the default coordinate system described by the default basis of vectors $x_{1}, x_{2}, x_{3}$ to the new built one with the basis described by $x_{1}^{\prime}, x_{2}^{\prime}, x_{3}^{\prime}$. At the end of the day, the rigid transformation is a rotation. In the new coordinate system all the projection points are on the same plane which is parallel to the $\vec{x}, \vec{y}$ plane, a $2 \mathrm{D}$ space. By ignoring the same $\mathrm{Z}$ coordinate of the points in the new built coordinate system, the system transforms the projection points in the $3 \mathrm{D}$ space to $2 \mathrm{D}$ space. At this point, the points only have $\mathrm{X}, \mathrm{Y}$ coordinates and are in a 2D coordinate system. 
Measure the wound area (step 4): Points enclosed in the outlined drawing are sparsely distributed in the $2 \mathrm{D}$ coordinate system. To calculate the wound area, these 2D points must be connected to from a closed region first. The system needs closed regions to calculate the wound's area. The system achieves this by placing these points in an image followed by morphological transformations. The positions of the points in the image is based on the $\mathrm{X}, \mathrm{Y}$ coordinates of the points. But the $\mathrm{X}, \mathrm{Y}$ coordinates of some points may be negative or very large, indicating that these coordinates cannot be used directly as the positions of the points in the image. The system first translates the points near the origin and in the first quadrant of the $2 \mathrm{D}$ coordinate system while preserving the relative position. The system achieves the translation by shifting the points by $x_{\min }$ units along the negative direction of $\mathrm{X}$ axis and by $y_{\min }$ units along the negative direction of $\mathrm{Y}$ axis; $x_{\min }$ and $y_{\min }$ are the minimum of the $\mathrm{X}$ and $\mathrm{Y}$ coordinates of all the points, respectively. At this point, the coordinates of the points can be used directly as the positions of the points in the image. Then, the system creates an image with $x_{\max }-x_{\min }$ as height and $y_{\max }-y_{\min }$ as width and places the points in the image using the points' coordinates as the position of the points; here $x_{\max }$ and $y_{\max }$ are the maximum of the $\mathrm{X}$ and $\mathrm{Y}$ coordinates of all the points, respectively. The region encompassed by the points is the area of the wound. With the help of OpenCV, the proposed approach performs the morphological operation dilation on the image, a matrix of ones as the structuring element. After dilation, gaps between the points are filled and the points are connected. Further, the system performs Zhang and Suen thinning operation on the image and the skeleton of the structure is found; see Fig.13. Thanks to OpenCV, the approach finally obtains the main contour of the structure and counts pixels within the contour as the area of the wound. 
Measure the wound volume (step 5): To calculate the wound volume, the system partitions the cavity of the wound into small individual cubic based on the pixels in the color image. The encompassed region by the drawing contour is the area of interest and composed of pixels in the color image. For each pixel, there is one corresponding point in the 3D space and the proposed approach maps the pixel itself and three neighbors (the right, lower and the lower right sub-pixels of this pixel) from color image to depth image and further to points in the point cloud to get four corresponding points by following the above procedure. Further, the system obtains the projection points of the four mapped points on the fitting plane obtained above. The four projection points form a quadrilateral. The system iterates all the pixels and sums up the area of all the quadrilaterals as the area of the wound region. The four mapped points in the point cloud and four corresponding projection points on the fitting plane are the eight vertices of the cubic that is composed of two triangular prisms. For each triangular prism, the system calculates the volume of the triangle prime by multiplying the area of the base of the triangular prism with the height, which is the average of the distance of the three mapped points to the fitting plane. The sum of the volume of the two triangular prisms is the volume of the cubic. The approach iterates all the pixels and sums up the volume of all the cubic as the volume of the wound. 


\section{EXPERIMENT AND RESULTS}

In this section we aim to evaluate the accuracy of the proposed system. In particular, we are interested in measuring the accuracy of each of the steps discussed in Section 2.22 that comprises the proposed system.

To evaluate the proposed system, we use two physical 3D models. The first one is a planar object, which is an A4 paper pasted on a cardboard. We call this the paper model. This planar object will help us determine the accuracy of the system in measuring areas. The second model is a plastic 3D wound model. This wound model is commercially available mainly for training nurses; the goal of these models is to replicate realistic scenarios. These wound models are manufactured by KCI company $^{3}$; Fig.7 shows an image of the wound model.
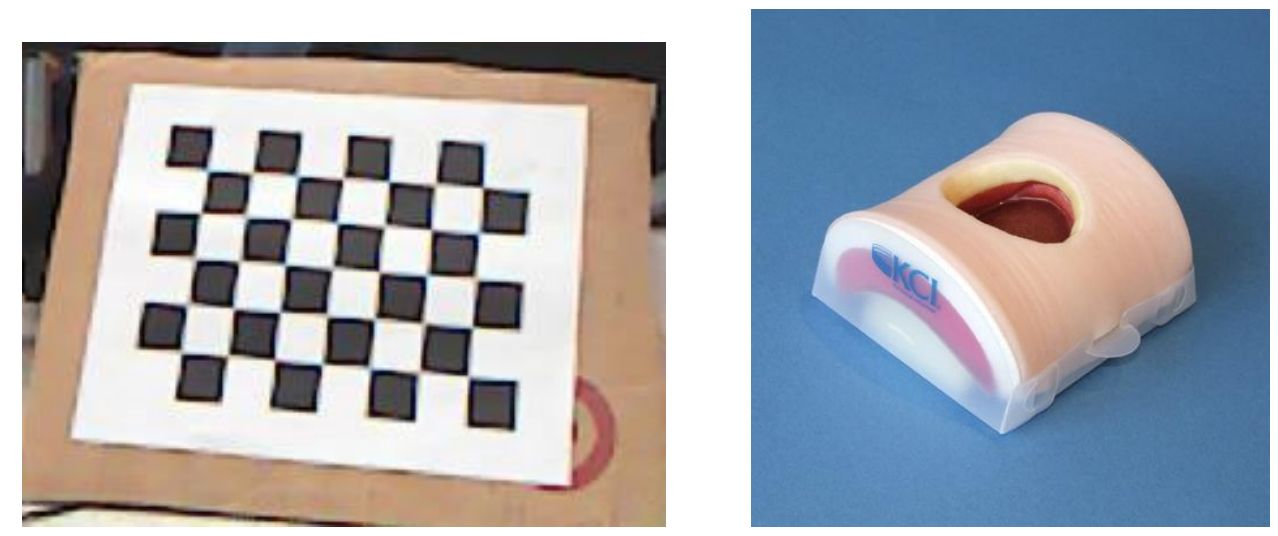

Figure 7: Paper model (left) and wound model (right). The left image shows the paper model and the right one shows the wound model.

To carry out these experiments, we place either the model in front of the Kinect and we adjust the position and orientation of the model to make sure that the entire model within the field of view of the Kinect. Then, we draw the contour along boundary of the model in the color or RGB image. Subsequently, the proposed system

\footnotetext{
${ }^{3}$ www.kci.com
} 
maps the contour into $3 \mathrm{D}$ space and fits a plane to these $3 \mathrm{D}$ points to obtain the projection of the points in the contour on the estimated plane; these points are useful to calculate the area. Further, the proposed system estimates a rigid transformation, and maps the projected points to facilitate the area and volume estimation. Based on the projected points, the system creates an image and performs morphological transformations on the created image. Finally, the proposed system calculates the area and volume of the model as described in the previous Section. For each step of the proposed approach, we record the internal data to analyze and evaluate the system.

The evaluation of each component in the proposed system consists of four experiments. In the first experiment we evaluate the fitting plane process. To do this, we first tune the distance threshold parameter for RANSAC utilizing the paper model. Then, we evaluate the output of RANSAC, i.e., the estimated plane. In experiment 2, we evaluate the estimation of the rigid transformation that transforms points in order to facilitate the area and volume calculation. In experiment 3 , we evaluate the effects of dilation and thinning operation on the created image after rigid transforming points. In experiment 4 , we systematically analyze the estimated area and volume of the models. This experiment reveals the accuracy of the proposed system. The four experiments are fully described below.

\subsection{Experiment 1: Fitting plane verification}

To calculate the wound area and volume, the proposed system fits a plane using RANSAC, which is the foundation for the wound assessment and crucial for the system. The fitting plane is the output of RANSAC. We first tune the parameters of RANSAC to form a solid foundation. There are two parameters that need to be tuned for the system. These parameters are the distance threshold and maximum number of 


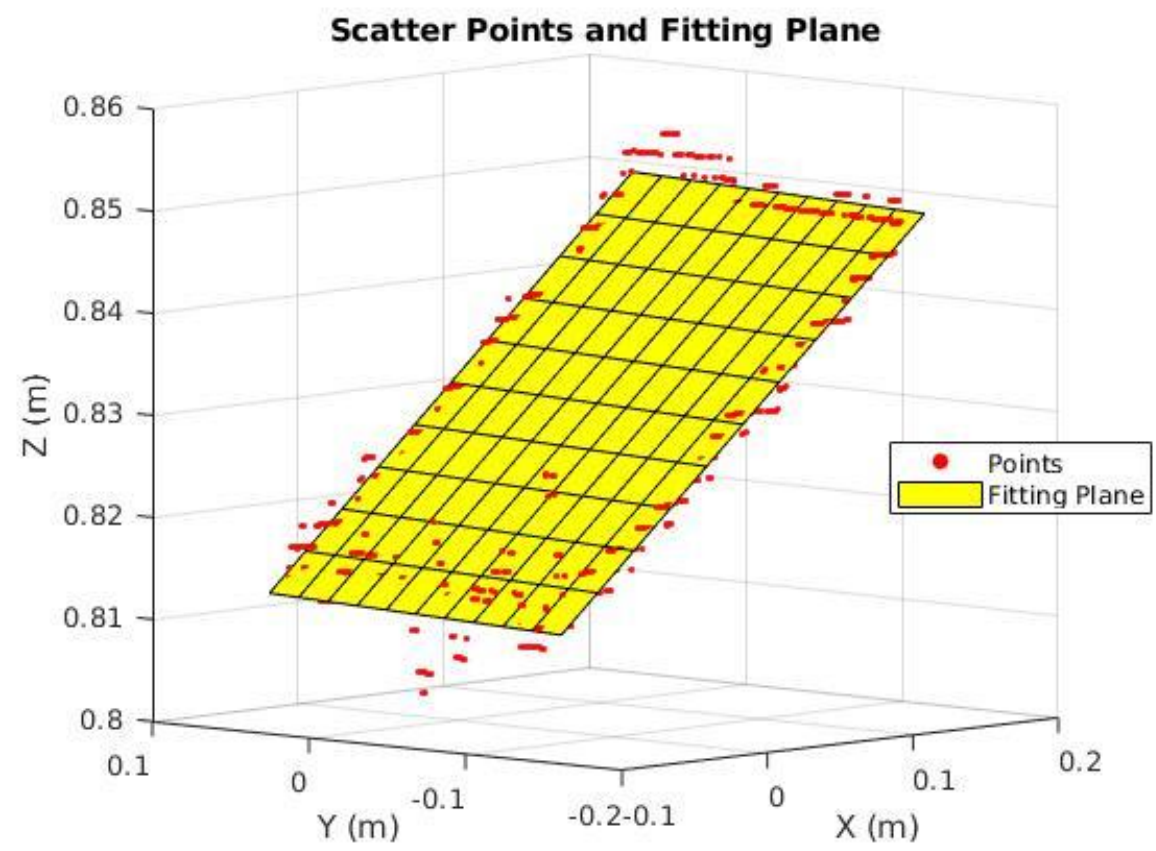

Figure 8: The outline wound points and the fitting plane for the paper model. As illustrated in the figure, some points (red) are off the fitting plane.

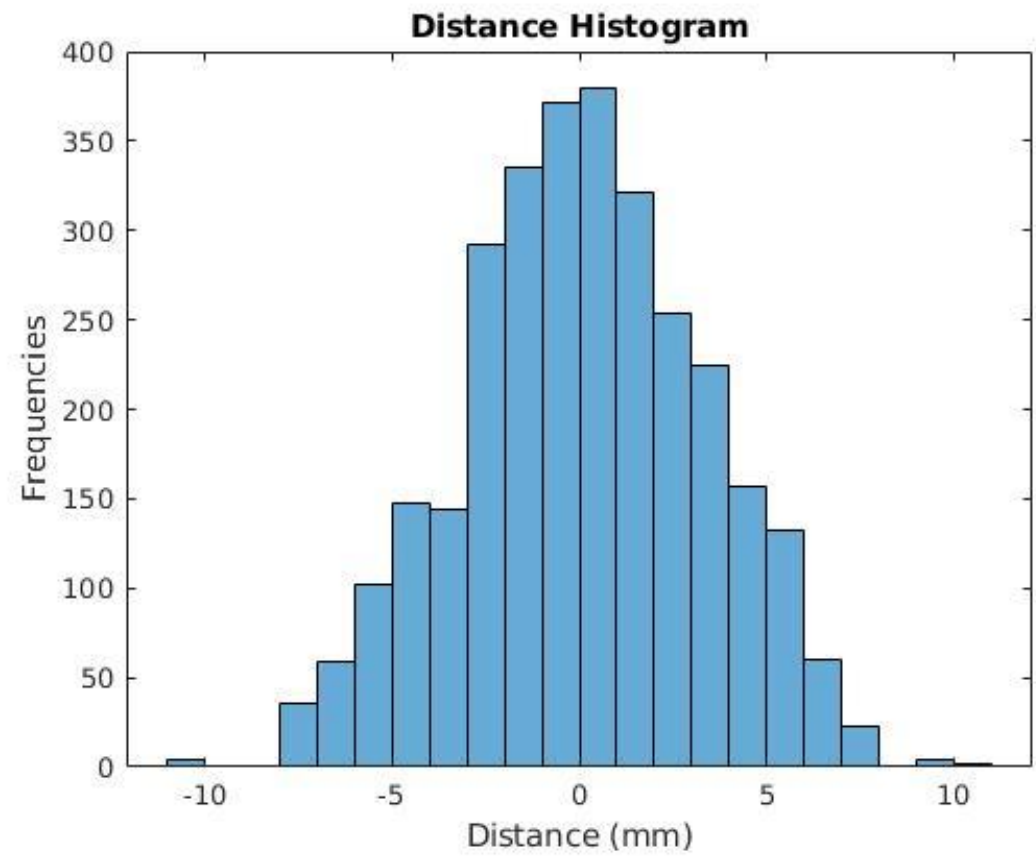

Figure 9: The histogram of the distance of the outline points to the fitting plane. As illustrated in the figure, the distribution of the distance is close to a normal distribution.

iterations. Since most of the mapped outlined wound boundary points are inliers or points that fit the estimated plane well, the system sets the maximum number of iterations to be 200 , which is more than enough. On the other hand, setting the distance threshold is more complicated. If such threshold is too large, then all the 
potential fitting planes tend to be ranked equally. On the other hand, when the distance threshold is too small, the estimated fitting plane tends to be unstable. We estimate the right threshold by fitting a plane using the paper model. Since the paper model is a perfect planar model, all the boundary points of the model should be the inliers of the fitting plane, and the distance of the boundary points can be analyzed to find its distribution. The paper model is placed in front of the Kinect and we draw the contour along the boundary of the paper; see Fig. 5 for an illustration of this setting. The proposed system maps the contour into 3D space and fits a plane for the paper. As shown in Fig.8, the border points (shown in red) won't always fall on the fitting plane (shown in yellow), even for a simple and perfect planar model due to Kinect noise. The system calculates the distance of the points to the fitting plane and plots the distribution of the distance, which is close to a normal distribution around zero; see Fig.9. The standard deviation (STD) of the distance is $3.2 \mathrm{~mm}$. To make sure that the inlier points of fitting plane have a 0.95 probability falling within the distance threshold, the system sets the distance threshold to be $3.2 * 2=6.4 \mathrm{~mm}$. When setting the distance threshold to be $6.4 \mathrm{~mm}, 95.64 \%$ of the border points are inliers for the paper model.

Table 1: The standard deviation of the distance of mapped border points to the fitting plane and the ratio of inlier points of the fitting plane for the wound model. Although there are discrepancies in the inlier ratio, the inlier ratio is still high (above 80\%). The standard deviation of the distance is also very small, less than $3.5 \mathrm{~mm}$.

\begin{tabular}{|c|c|c|c|c|c|}
\hline Experiment & 1 & 2 & 3 & 4 & 5 \\
\hline STD $(\mathrm{mm})$ & 3.3864 & 3.3952 & 3.3986 & 3.4866 & 3.4478 \\
\hline Inlier Ratio & 0.8261 & 0.8509 & 0.8820 & 0.7950 & 0.8820 \\
\hline
\end{tabular}


Table 2: The average and standard deviation of the output volume and area for the wound model. For each contour, the standard deviation is only about $1 \%$ of the average for the wound area and 5\% for the wound volume, indicating that the system itself is robust. For different contours of the frontal orientation, there is clear difference due to different contour drawings covering different area. For different orientations, there is also discrepancy among the wound area and volume. But the discrepancy between different orientations is comparable to the difference among the same orientation (frontal orientation). For the wound model, the ground truth area is $3,268 \mathrm{~mm}^{2}$, which means that the system has $93 \%$ or more accuracy for the wound model area measurement and the wound model's ground truth volume is obtained as $58,000 \mathrm{~mm}^{3}$, which means that the system has $60 \%$ or more accuracy for the wound model volume measurement.

\begin{tabular}{|c|c|c|c|c|c|}
\hline Contour & 1 & 2 & 3 & 4 & 5 \\
\hline Orientation & Frontal & Frontal & Frontal & Left & Right \\
\hline Volume Average & 35768.16 & 43950.76 & 39197.48 & 35350 & 35350 \\
\hline Volume STD & 1900.451 & 1856.479 & 2027.985 & 1757.316 & 1927.329 \\
\hline $\begin{array}{c}\text { Volume } \\
\text { STD/Average }\end{array}$ & 0.053132 & 0.04224 & 0.04224 & 0.049712 & 0.046557 \\
\hline Area Average & 3067.58 & 3164.245 & 3224.22 & 3371.6 & 3056.98 \\
\hline Area STD & 31.66798 & 33.23122 & 37.92328 & 27.88607 & 31.13925 \\
\hline $\begin{array}{c}\text { Area } \\
\text { STD/Average }\end{array}$ & 0.010323 & 0.010502 & 0.011762 & 0.008271 & 0.010186 \\
\hline
\end{tabular}

Here we aim to evaluate the fitting of the plane. The experiment evaluates the fitting plane by checking whether it satisfies the hypotheses that the selected skin region is nearly flat in healthy condition, and the mapped border points of the wound should fall on a fitting plane or closed enough to the fitting plane. The experiment evaluates the fitting plane using the wound model. The operator draws the contour of the wound model in the color image and the experiment uses the same contour as the input of proposed system and run it five times. Each time, the experiment calculates the distance of the mapped border points to the fitting plane. The standard deviation of 

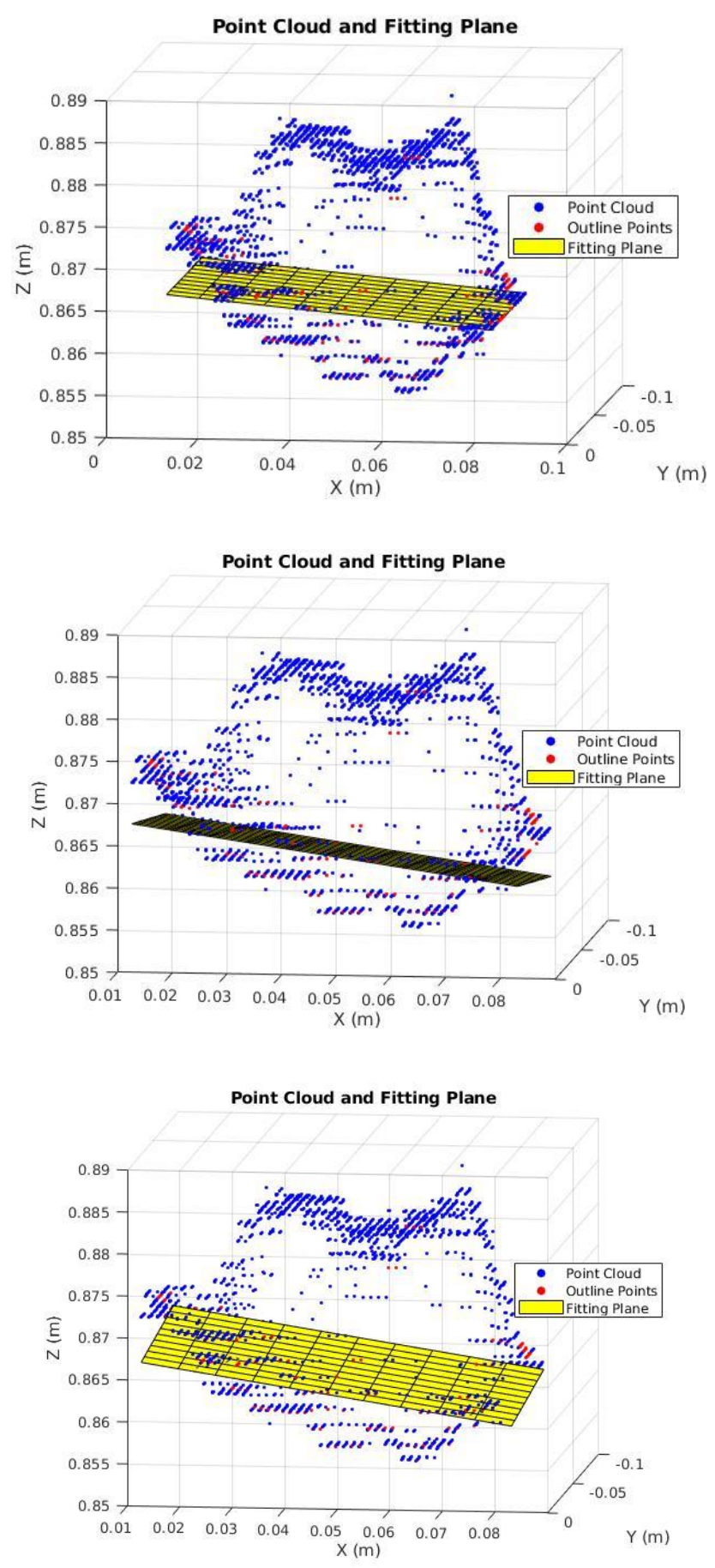

Figure 10: The fitting plane and the point cloud of the wound model. The system generates three different fitting planes (yellow) in the point cloud (blue) with the same outline points (red). As illustrated in the figure, although the three planes are different, they are very similar to each other. the distance and the ratio of inlier points (total points within distance threshold / total mapped border points) are listed in Table 1. Although there are discrepancies in the inlier ratio, the inlier ratio is still high (above 80\%). The standard deviation of the distance is also very small, less than $3.5 \mathrm{~mm}$. The high liner ratio and less standard deviation indicates that most of the mapped points are on the fitting plane or close enough to the fitting plane, which proves the hypothesis. Although the inlier ratio is very high, there are still discrepancies among them, indicating that the fitting plane is not unique for the same contour. RANSAC may generate different planes with 
the same input. As illustrated in Fig.10, the system generates different planes with the same input. Will this affect the wound assessment? The evaluation experiments also evaluate this. We draw five contours along the wound boundary of the wound model. For each contour, the experiment uses the same contour as the input of the proposed system to run fifty times and record the output wound volume and area to calculate the average and standard deviation. As illustrated in Table 2, for each contour, the standard deviation is only about $1 \%$ of the average for the wound area and $5 \%$ for the wound volume, indicating that although the system may generate different fitting planes, these estimated planes are similar, and the discrepancy only slightly affects the wound assessment.

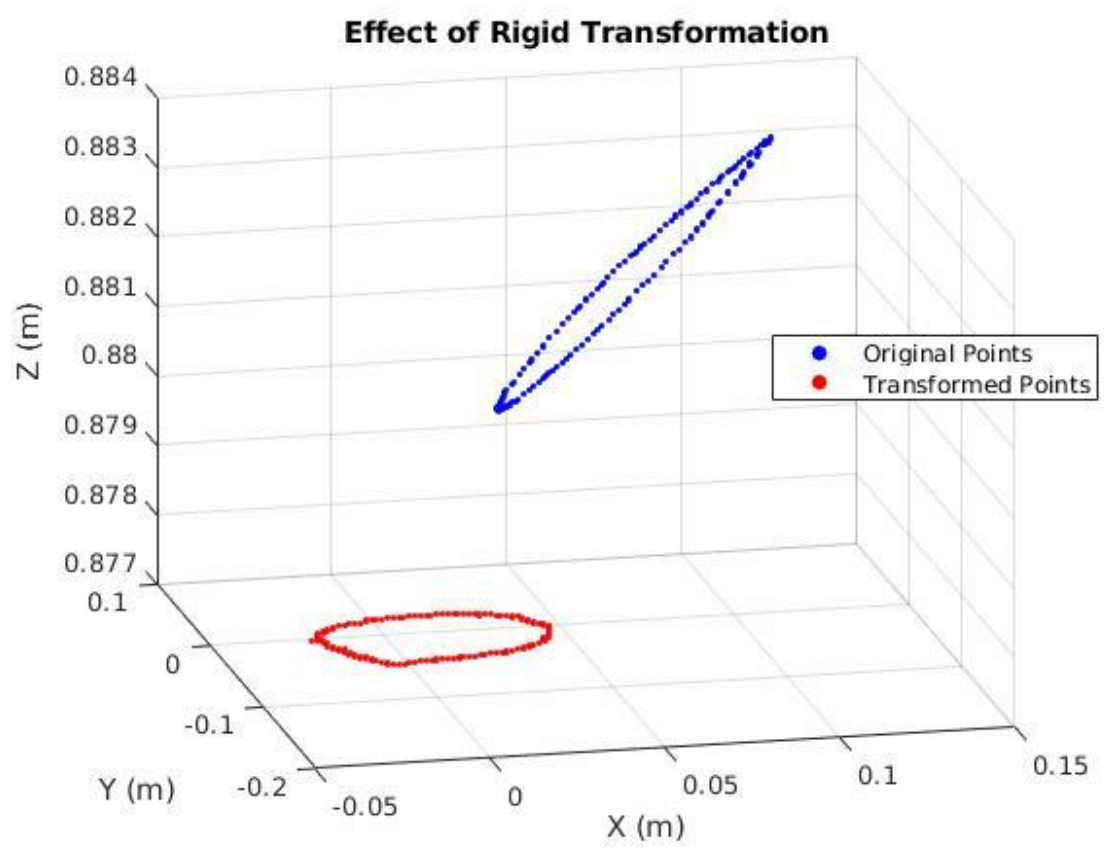

Figure 11: The effect of rigid transformation. The original points (blue) are scatter in the 3D space of the default coordinate system. After the rigid transformation, the transformed points (red) are in a plane parallel to the $\mathrm{X}, \mathrm{Y}$ plane. 


\subsection{Experiment 2: Rigid transformation verification}

Here we aim to evaluate the estimation of the rigid transformation. After the system projects the mapped border points onto the fitting plane, the region encompassed by the projection points is the wound area. To facilitate the calculation of the wound area, the proposed system transforms the projection points from 3D space to 2D space by transforming the projection points from the default coordinate system to a new coordinate system with the normal vector of the fitting plane as the $\mathrm{Z}$ axis. To verify the transformation, the evaluation experiments plot the original and transformed projection points in the same coordinate system. As illustrated in Fig.11, the original projection points (red) are scattered in the 3D space, which is difficult to calculate the area in such 3D space. After the rigid transformation, the transformed points (blue) are in a plane parallel to the $\mathrm{X}, \mathrm{Y}$ plane. Since the transformed points are in the $\mathrm{X}, \mathrm{Y}$ plane and have the same $\mathrm{Z}$ coordinates, the system just ignores the $\mathrm{Z}$ coordinate. At this stage, the points only have $\mathrm{X}, \mathrm{Y}$ coordinates, which indicates that the original projection points are transformed from 3D space to 2D space and confirms the validity of the rigid transformation.

\subsection{Experiment 3: Morphological transformation verification}

Although the rigid transformation transforms the points into $2 \mathrm{D}$ space and the system places the points in an image based on the points' coordinates, these points are still disconnected in the image, as shown in Fig.12. The system adopts morphological transformation to solve this issue. Here we aim to evaluate the effect of the morphological transformation and verify that points are connected.

To evaluate morphological transformation, the experiment displays the image after dilation operation and the image after thinning operation to assess the effect of the 
morphological transformation. As shown in Fig.13, dilation operation connects all the points but the boundary becomes very thick. After the thinning operation, the thick boundary becomes thin and only the skeleton of the boundary is left on the image. The left skeleton matches the shape of the original scattered points, except that the skeleton is connected, which confirms that the morphological transformation successfully fulfills its duty.
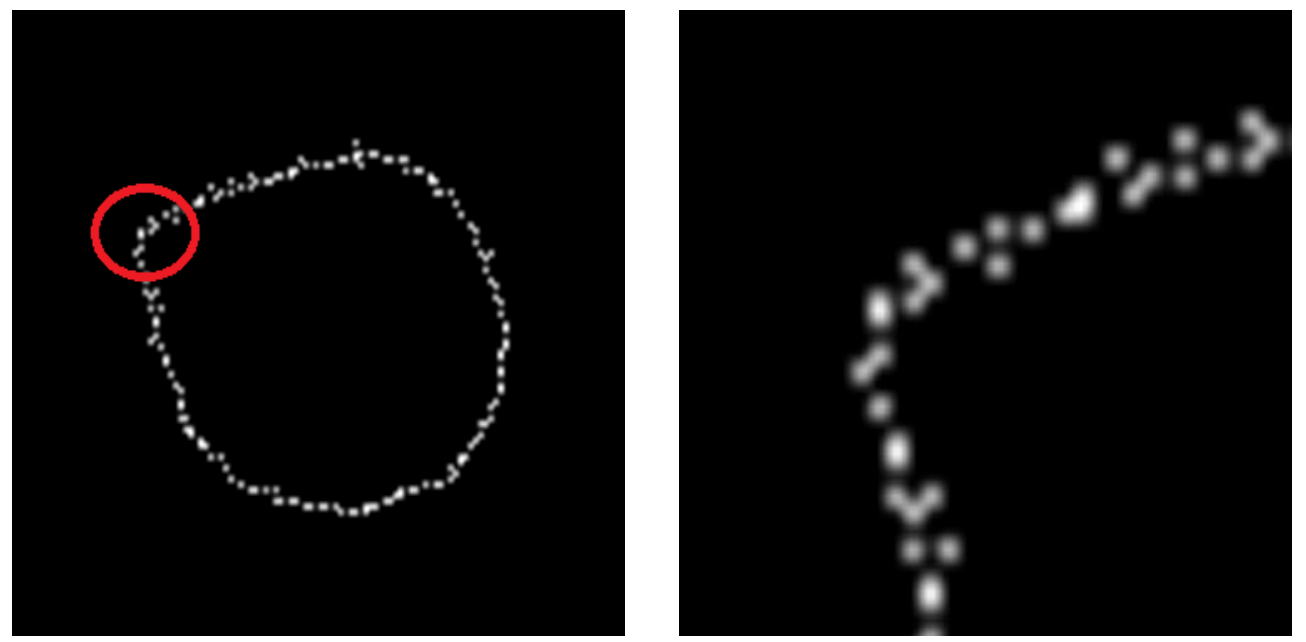

Figure 12: The disconnected projection points. The left image shows the projected points in the metric image and the right is the enlargement of the circle region (red) in the left. As illustrated in the image, although some points are connected, the rest are disconnected in the image.
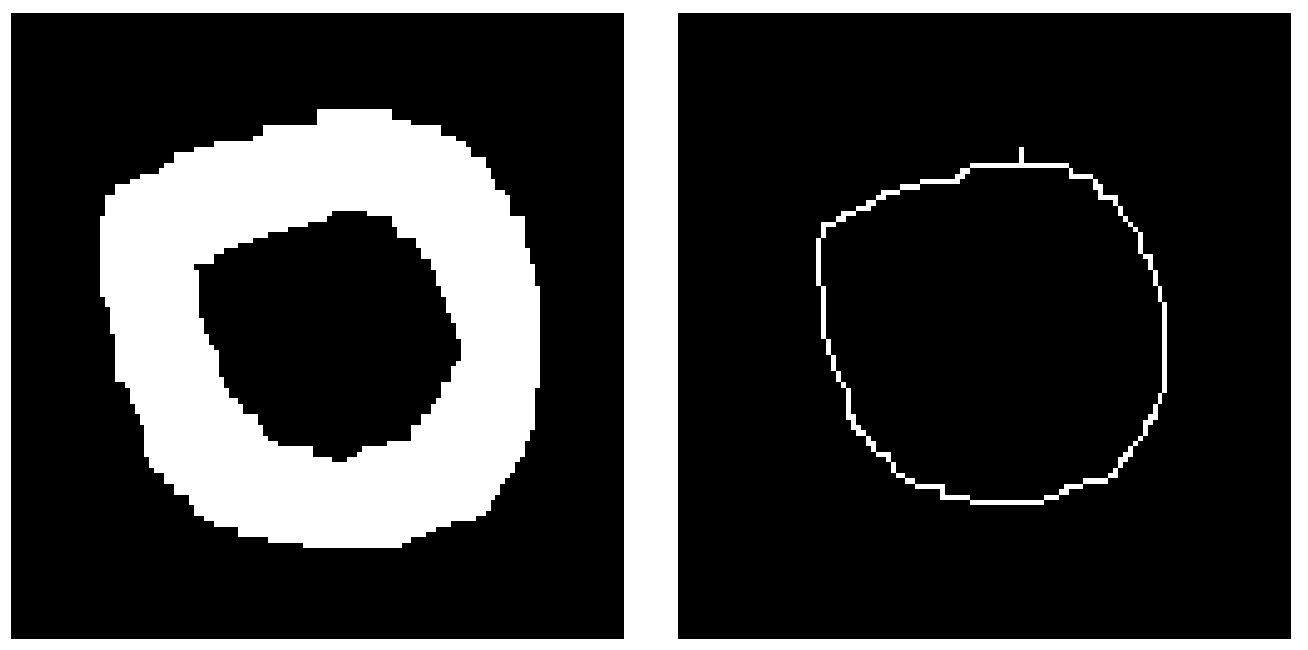

Figure 13: The effect of dilation and thinning operation. The disconnected points are connected, and the boundary becomes thick after dilation operation (left). After the thinning operation, the skeleton of the boundary is left on the image (right). 


\subsection{Experiment 4: Robustness and measurement accuracy verification}

To consistently monitor the healing process, the system should be robust to viewpoint and noisy inputs. We first evaluate the robustness of the system. For the same wound, different clinicians should get same or close wound assessment. To evaluate the robustness of the system, the wound model is placed in three different orientations. First, we place the wound model facing to the Kinect (frontal orientation), then slightly rotate the orientation to the left and right. We draw three contours for the frontal orientation and one contour for left and right orientation. For each contour, the experiment uses the same contour as the input of the proposed system to run fifty times and record the output wound volume and area to calculate the average and standard deviation. As shown in Table 2, for each contour, the standard deviation is only about $1 \%$ of the average for the area and $5 \%$ of the average for the volume. Note that the same contour has only a small deviation for the wound area and volume, which indicates that the system itself is robust. However, for

different contours of the frontal orientation, there is clear difference between the calculated wound area and volume due to the error introduced by the operator. Recall that the user draws the contour with a pointer (e.g., mouse) on the screen, and this introduces noise and variation. Different contour drawings may cover a different area and the error can be easily introduced here. For different orientations, there is also discrepancy among the wound area and volume. But the discrepancy between different orientations are comparable to the difference among the same orientation (frontal orientation), indicating that the system is also robust to the orientation of the wound model if the entire wound model is under the view of the Kinect. 
Table 3: The area measurement for paper model.

\begin{tabular}{|c|c|c|c|c|c|c|}
\hline Experiment & 1 & 2 & 3 & 4 & 5 & Average \\
\hline Area & 44126 & 44908 & 43961 & 44921 & 46908 & 44964 \\
\hline
\end{tabular}

Although the system is robust, we also aim to evaluate the measurement accuracy of the system. The experiment measures the area of the paper model five times and the area is listed in Table 3. The paper model's ground truth area is $210 \times 297 \mathrm{~mm}$, which is $62,370 \mathrm{~mm}^{2}$. The average estimate of the area that the system computes is 44964, which means that the system achieves $72.1 \%$ accuracy for the planar model. For the wound model, the ground truth area is obtained using the trace paper and is $3,268 \mathrm{~mm}^{2}$, which means that the system has $93 \%$ or more accuracy for the wound model area measurement. Through filling the wound cavity with plasticine and measuring the volume of the plasticine, the wound model's ground truth volume is obtained as $58,000 \mathrm{~mm}^{3}$, which means that the system has $60 \%$ or more accuracy for the wound model volume measurement. The volume measurement has lower accuracy than the area due to the additional dimension in calculating the volume. The difference between the wound model and paper model is due to the model size and shape. The paper is planar and much bigger, so the contour is easy to define. For the small wound model, the error can be easily introduced because it is not planar and noise coming from the Microsoft Kinect sensor and human input. Considering that the Kinect is not perfect, overall the measurement accuracy of the proposed system is acceptable. 


\section{CONCLUSIONS}

This report presented a system that uses inexpensive RGB-D cameras to measure wound area and volume. The report introduces a simple yet effective methodology to estimate these metrics, which can be useful to monitor the healing progress of a patient requiring wound treatments. As the experiments show, the proposed system achieves a good accuracy despite using inexpensive and noisy imaging sensors. The proposed methodology presented in this report still can be useful with a higher-end depth camera sensor, which can greatly improve the estimates and deliver a more accurate measurement of area and volumes of a wound. 


\section{REFERENCES}

1. Tallman, Phillip, et al. "Initial rate of healing predicts complete healing of venous ulcers." Archives of dermatology 133.10 (1997): 1231-1234.

2. Kantor, J., and D. J. Margolis. "A multicentre study of percentage change in venous leg ulcer area as a prognostic index of healing at 24 weeks." British Journal of Dermatology142.5 (2000): 960-964.

3. Gethin, Georgina. "The importance of continuous wound measuring." WOUNDS UK 2.2 (2006): 60.

4. Filko, Damir, Robert Cupec, and Emmanuel Karlo Nyarko. "Detection, reconstruction and segmentation of chronic wounds using Kinect v2 sensor." Procedia Computer Science90 (2016): 151-156.

5. Langemo, Diane, et al. "Measuring wound length, width, and area: which technique?." Advances in skin \& wound care 21.1 (2008): 42-45.

6. Chang, Angela Christine, Bronwyn Dearman, and John Edward Greenwood. "A comparison of wound area measurement techniques: visitrak versus photography." Eplasty 11 (2011).

7. Hartley, Richard, and Andrew Zisserman. Multiple view geometry in computer vision. Cambridge university press, 2003.

8. Yang, Lin, et al. "Evaluating and improving the depth accuracy of Kinect for Windows v2." IEEE Sensors Journal 15.8 (2015): 4275-4285.

9. Izadi, Shahram, et al. "KinectFusion: real-time 3D reconstruction and interaction using a moving depth camera." Proceedings of the 24th annual ACM symposium on User interface software and technology. ACM, 2011. 
10. Henry, Peter, et al. "RGB-D mapping: Using Kinect-style depth cameras for dense 3D modeling of indoor environments." The International Journal of Robotics Research 31.5 (2012): 647-663.

11. Xiao, Jianxiong, Andrew Owens, and Antonio Torralba. "Sun3d: A database of big spaces reconstructed using sfm and object labels." Computer Vision (ICCV), 2013 IEEE International Conference on. IEEE, 2013.

12. Fischer, M. A., and R. C. Bolles. "A paradigm for model-fitting with applications to image analysis and automated cartography." (1981).

13. Derpanis, Konstantinos G. "Overview of the RANSAC Algorithm." Image Rochester NY 4.1 (2010): 2-3

14. Yang, Michael Ying, and Wolfgang Förstner. "Plane detection in point cloud data." Proceedings of the 2nd int conf on machine control guidance, Bonn. Vol. 1. 2010.

15. Fujiwara, Tomofumi, Tetsushi Kamegawa, and Akio Gofuku. "Plane detection to improve 3D scanning speed using RANSAC algorithm." Industrial Electronics and Applications (ICIEA), 2013 8th IEEE Conference on. IEEE, 2013.

16. Deschaud, Jean-Emmanuel, and François Goulette. "A fast and accurate plane detection algorithm for large noisy point clouds using filtered normals and voxel growing." Proceedings of the 5th International Symposium on 3D Data Processing, Visualization and Transmission (3DPVT'10). 2010.

17. http://homepages.engineering.auckland.ac.nz/ pkel015/SolidMechanicsBooks /Part_III/Chapter_1_Vectors_Tensors/Vectors_Tensors_05_Coordinate_Transf ormation_Vectors.pdf

18. Burger, Wilhelm, and Mark J. Burge. Digital image processing: an algorithmic 
introduction using Java. Springer, 2016.

19. Zhang, T. Y., and Ching Y. Suen. "A fast parallel algorithm for thinning digital patterns." Communications of the ACM 27.3 (1984): 236-239. 